\title{
Memory Storage as a Function of Arousal and Time with Homogeneous and Heterogeneous Lists
}

\author{
Edward L. WALkeR ${ }^{1}$ and Robert D. TARTe \\ University of Michigan, Ann Arbor, Michigan
}

This study is basec on a general theory which interrelates perseverative consolidation, action decrement, and arousal (Walker, 1958). The relevant propositions of the theory are the following. (1) The occurrence of any psychological event, such as an effort to learn an item of a paired-associate list, sets up an active, perseverative trace process which persists for a considerable period of time. (2) The perseverative process has two important dynamic characteristics: (a) permanent memory is laid down during this active phase in a gradual fashion; $(b)$ during the active period, there is a degree of temporary inhibition of recall, i.e., action decrement (this negative bias against repetition serves to protect the consolidating trace against disruption). (3) High arousal during the associative process will result in a more intensely active trace process. The more intense activity will result in greater ultimate memory but greater temporary inhibition against recall.

The major documentation of this theory has been with studies of the behavior of rats (Walker, 1956; Walker, 1958; Walker and Motoyoshi, 1962; Walker and Paradise, 1958) but two studies in human verbal learning which report results which appear to confirm the theory have been reported by Kleinsmith and Kaplan (1963a, b). One study employed eight words as stimulus items

\footnotetext{
1 This study was supported by grant no. M-4239 to the senior author from the United States Public Health Service, National Institutes of Health.
}

and the other used six low-association nonsense syllables. Single digits were the response items in both studies, and in both a single learning trial was followed by a single recall trial. The interval between learning and recall was varied from $2 \mathrm{~min}$. to one week. In both studies skin resistance was measured as a response to each stimulus item during learning. In each case it was found that associations learned in the presence of low arousal, i.e., little change in skin resistance, showed high immediate recall which then fell to a low value at the end of a week. Items associated in the presence of a large skin-resistance change, and thus under high arousal, showed poor immediate recall but high recall at $45 \mathrm{~min}$. to one week.

The present study consists of a replication of the Kleinsmith and Kaplan studies and a test of whether the results can be obtained with homogeneous lists of high- and lowarousal words.

\section{METhoD}

Subjects. Seventy-two women were selected from the elementary course in Psychology. Their service as $S$ s was a requirement of the course.

Learning Materials. The stimulus materials for the high-arousal list consisted of the words: money, rape, slut, embrace, kiss, vomit, passion and sex. The stimulus materials for the low-arousal list were the words: white, pond, berry, flower, walk, pencil, glass, and carrot. The mixed or heterogeneous list was composed of a selection of four words from the high-arousal list and four from the low-arousal list. The response items were the digits 2 through 9 .

Design. The $72 \mathrm{Ss}$ were divided into 9 groups of 
$8 \mathrm{Ss}$ each. Three groups learned the high-arousal list, three learned the low-arousal list, and three learned the mixed list. The interval between learning and recall was $2 \mathrm{~min}$. for one group, $45 \mathrm{~min}$. for another, and one week for the third for each of the three lists. The $8 \mathrm{Ss}$ in each group each had a different order of presentation of the words so that each word appeared in each of the eight positions in the list for one $S$ each of the homogeneous-list groups. In the mixed-list groups, lists were composed of four high-arousal and four low-arousal items, and each of the high- and each of the lowarousal words appeared in four mixed lists. In these groups, high-arousal items appeared an equal number of times in each position in the list, but complete order control by word could not be accomplished with $8 \mathrm{Ss}$.

Skin Resistance. The technique employed for the measurement of skin resistance is essentially that developed by Lykken (1959). The electrode is zinc and the paste is zinc sulphate. The recorder is a Varian G-11-A modified to extend the range. The basic datum is the basal resistance level (BRL) in ohms. Arousal is measured in per cent deflection of BRL in the presence of the stimulus and is essentially the traditional GSR.

Procedure. The $S$ s were run individually. The electrodes were fastened to the first and third fingers of the right hand, and they were allowed to rest while the Varian recorder was calibrated. The materials were programmed in a slide projector set to expose a new slide every $4 \mathrm{sec}$. The first slide contained a word, the second a word and a digit, and the third and fourth slides consisted of four colored squares. The fifth slide contained the second word, etc. The $S$ was instructed to speak each word aloud, to speak both word and number, and to name the colors in any order. The color slides were interpolated into the list to permit $S$ to recover from the arousal produced by one stimulus item before the next was exposed.

Recall was carried out with an additional color slide substituted for the word-number slide. Thus $S$ was given $4 \mathrm{sec}$. to recall the number associated with the exposed word and then spent $12 \mathrm{sec}$. naming colors before he was asked to recall a second item. The order of presentation of the stimulus words during recall was random with respect to the order during learning. When recall was 2 min. after training, the recall sequence was begun immediately after the learning trial. If recall was to be delayed $45 \mathrm{~min}$., $S$ was kept in the room and was given a book of New Yorker cartoons to read during the interval. The $S$ s who were to recall in one week were released after the learning trial and told they were to return in a week at which time, "I'll have another game for you then."

\section{RESUlTS}

While there can be little question that the words in the high-arousal list should be emotion-producing on a priori grounds, and that the words in the low-arousal list should be emotionally innocuous, the actual BRL deflections produced by the words in the two lists show considerable overlap as may be seen in Table 1. It may also be noted

TABLE 1

Mean Per Cent BRL Deflection in Presence of STIMULUS WORDS

\begin{tabular}{lcc}
\hline Words & $\begin{array}{c}\text { In } \\
\text { homogeneous } \\
\text { list }\end{array}$ & $\begin{array}{c}\text { In } \\
\text { heterogeneous } \\
\text { list }\end{array}$ \\
\hline High arousal words & & \\
Rape & 7.41 & 10.41 \\
Vomit & 5.51 & 9.55 \\
Kiss & 5.47 & 5.78 \\
Money & 5.13 & 4.44 \\
Slut & 4.65 & 5.47 \\
Passion & 3.51 & 3.08 \\
Embrace & 3.09 & 4.83 \\
Sex & 2.34 & 5.94 \\
$\quad$ Mean & 4.64 & 6.19 \\
\hline Low arousal words & & \\
Glass & 4.62 & 5.86 \\
Pond & 4.55 & 5.54 \\
Flower & 4.05 & 1.98 \\
Carrot & 3.20 & .03 \\
Berry & 3.06 & .23 \\
Walk & 2.97 & 2.00 \\
White & 2.54 & 4.47 \\
Pencil & 1.12 & .87 \\
Mean & 3.26 & 2.62 \\
\hline
\end{tabular}

that the deflection produced by a word appears to depend in part upon the context. There is some tendency for low-arousal words to produce less arousal in the mixed list and the high-arousal words to produce higher deflections when they are in the mixed list. These data taken with the fact that Kleinsmith and Kaplan found functional differential arousal with low-association non- 
sense syllables, would suggest an interpretation that the per cent deflection in the presence of the stimulus was in part a function of the emotion-producing qualities of the stimulus item and in part a function of the effort associated with the act of attention itself.

Recall. The capacity to recall as a function of time for the three lists may be seen in Fig. 1. It is clear that the capacity to recall the numbers associated with the low-arousal words drops as a function of time. Furthermore, the capacity to recall numbers associated with high-arousal items starts slightly lower, shows some drop at $45 \mathrm{~min}$., and then rises slightly at one week. The results are thus in the same pattern as those of Kleinsmith and Kaplan, but the magnitude of the effect is less, especially in immediate recall. The difference between the high- and low-

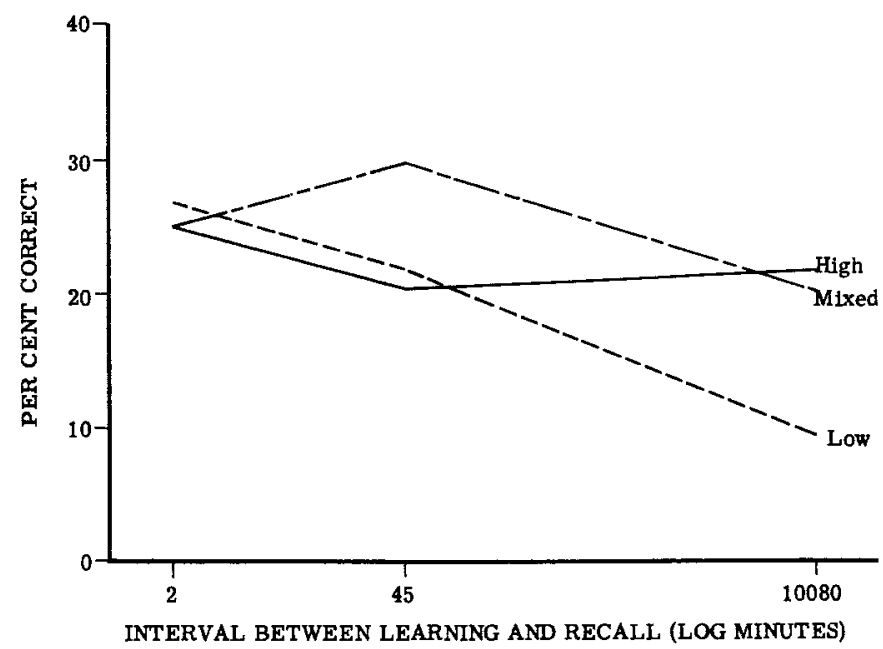

FIG. 1. Per cent recall for the two homogeneous and the mixed list as a function of time. High- and low-arousal words were selected on a priori grounds.

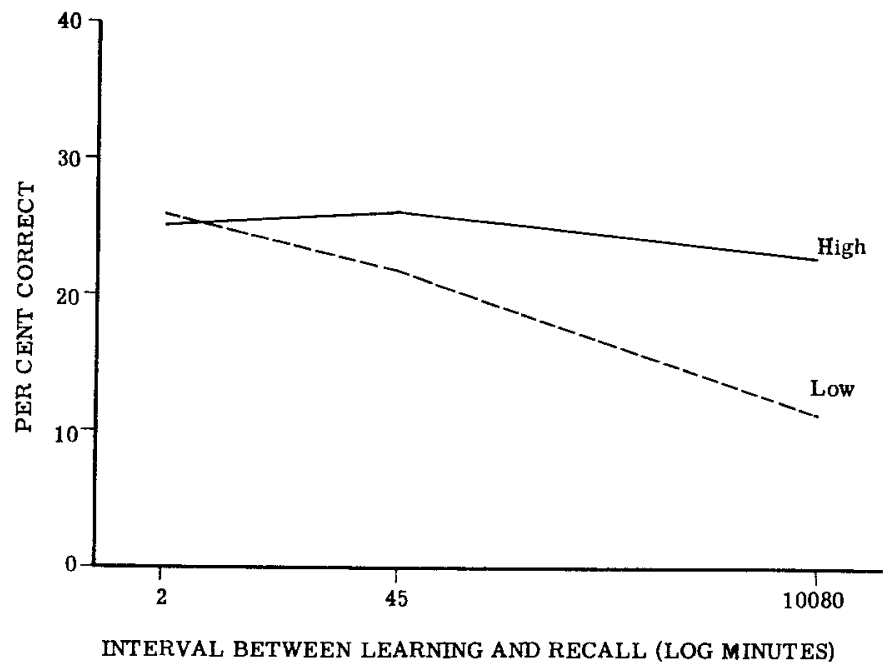

Frg. 2. Per cent recalled as a function of arousal when the items from the mixed list are combined with the appropriate homogeneous list. 
arousal curves is statistically significant. Figure 2 is a plot of the results when the items of the mixed list are combined with those of the homogeneous lists on the basis of their a priori identity as high- or low-arousal items.

Figures 3 and 4 contain two analyses similar to those presented by Kleinsmith and Kaplan. In Fig. 3 all data from the three lists are pooled. The 64 highest BRL deflections are labeled as high arousal, the 64 lowest are labeled as low arousal, and recall is then plotted as a function of arousal level during learning. In Fig. 4 the analysis is carried out within each of the $72 \mathrm{Ss}$, regardless of list, with the three words producing the highest deflection labeled as high, the three producing the lowest deflections labeled

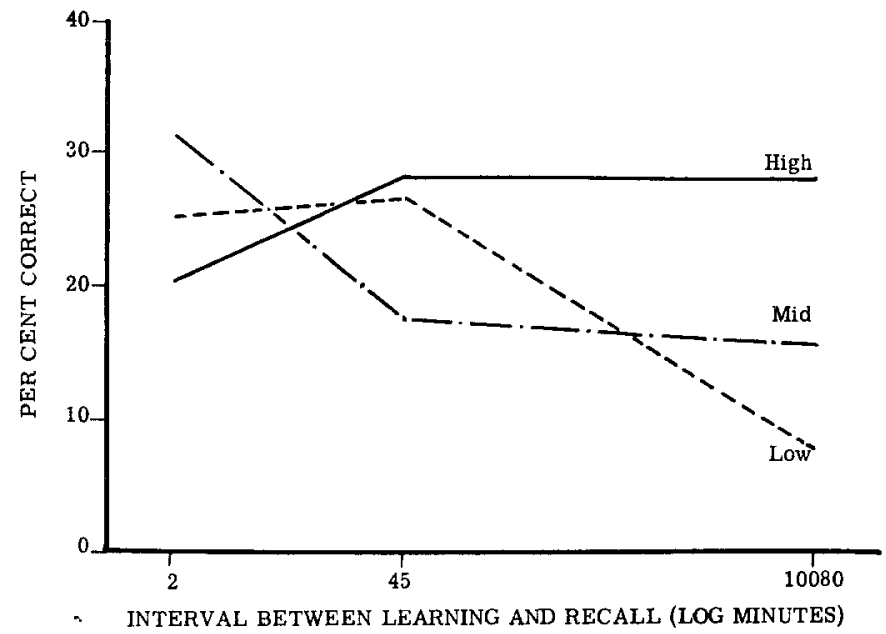

FIG. 3. Recall as a function of level of arousal during learning and time of recall when all items are pooled and categorized exclusively on the basis of the per cent BRL deflection during learning.

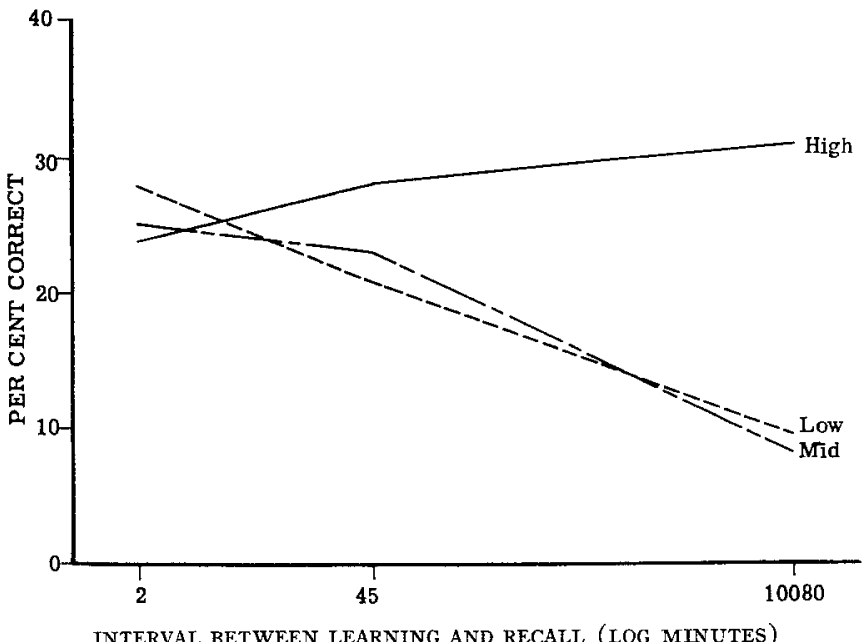

Frg. 4. Per cent recalled as a function of arousal during learning and time with analysis in terms of per cent BRL deflections to words within subjects. The three words producing the largest defiections are designated as high, and the three producing the lowest deflections are designated as low. 
as low, and the remaining two words labeled as mid. In both curves there is a close approximation to the Kleinsmith and Kaplan results although in these curves, the initial separation betwen high- and low-arousal items is considerably less than Kleinsmith and Kaplan obtained. In both figures the differences between the high- and low-arousal curves are statistically significant. Therefore, we may conclude that the general finding of Kleinsmith and Kaplan, that items learned under low arousal show high initial and low subsequent recall, while items learned under high arousal show lower initial recall and high ultimate recall, has been essentially replicated.

Serial Position. The serial position curve when recall is obtained at 2 min. shows a rather strong recency effect, while the serial position curve obtained at one week recall shows a strong primacy effect. Recent work on short term memory (see Melton, 1963) creates an expectation that the capacity to recall a learned item drops sharply during the first few seconds or minutes after learning. Figure 5 is a theoretical plot of the relative availability of the eight items of a pairedassociate list if recall is requested immediately after learning. Assuming a random recall order, the relative availability of an item for recall would be inversely related to the order of learning. Thus, the phenomena of short- term memory would lead to a prediction of a strong recency effect. The theory which is the basis for the present study would predict ultimate recall, in this case at one week, would vary directly with the arousal level during learning.

Figure 6 contains plots of the serial position curves at 2 -min. recall and 1-week recall along with the theoretical curve derived from consideration of the phenomena of shortterm memory, and a plot of BRL deflection as a function of serial position. It may be seen from the figures that the primacy effect in 1-week recall seems completely accounted for by the relative arousal by serial position. The correspondence between the theoretical and empirical curves for 2-min. recall is suggestive. It should be pointed out that the order of recall of items was random with respect to order of learning. An exact match between the theoretical and empirical curves for the 2-min. recall situation would be expected only where the order of recall was determined in such a manner as to make the time, or number of items, between learning and recall a controlled variable. Such a study remains to be carried out.

It should be noted that the primary relationships between arousal and time of recall are not essentially modified when serial position is controlled. This factor has been treated by Kleinsmith and Kaplan.
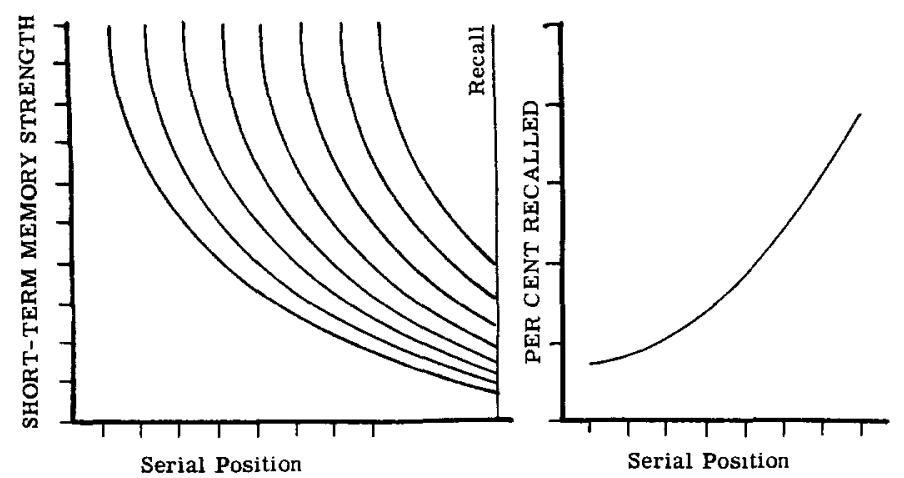

Fic. 5. Derivation of per cent recalled by serial position in terms of the phenomena of short-term memory for the situation in which recall immediately follows learning. 

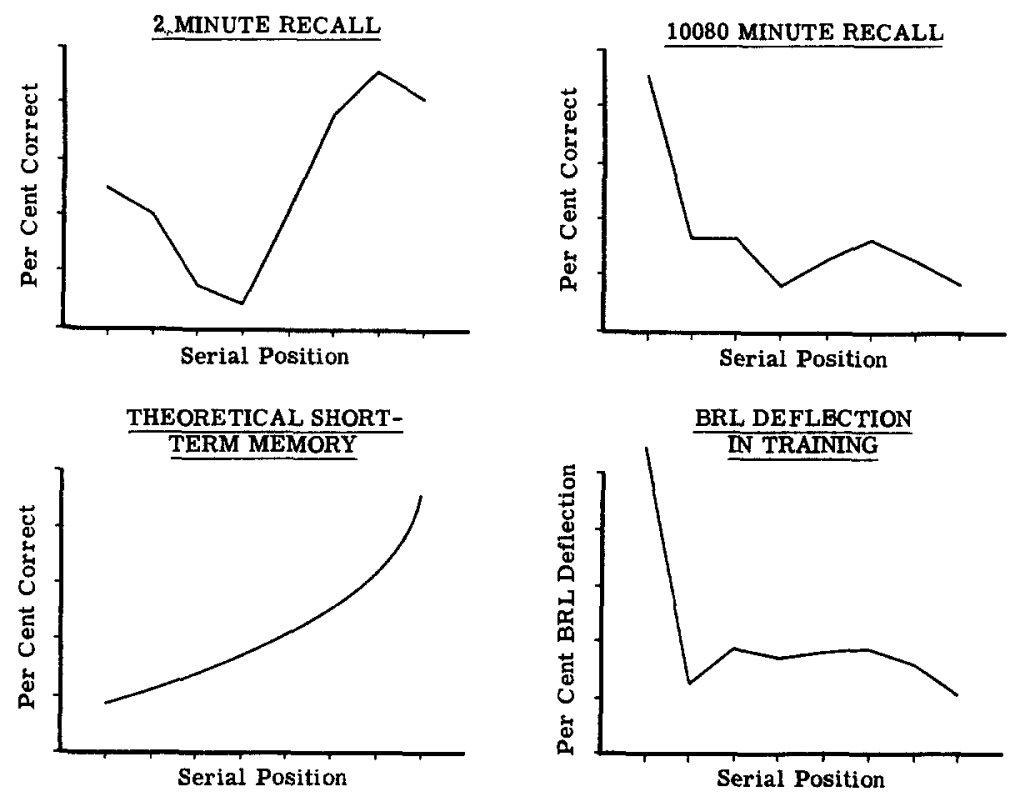

Fig. 6. Correspondence between predicted and obtained recency curves in 2-min. recall, and predicted and obtained primary curves in 10,080-min. (1-week) recall.

\section{SUMmaRY}

This study is one of a series designed to test a general theory of perseverative consolidation. The theory states the expectations that consolidation will be accompanied by a negative bias against repetition during its course and that an increase in arousal will produce an increase in the negative bias against repetition as well as an increase in the ultimate capacity to recall.

Seventy-two Ss were divided into 9 groups. Learning consisted of one trial with a list composed of 8 stimulus words and single digit numbers as response items. Three groups learned a high-arousal list, 3 groups a low-arousal list, and 3 learned a mixed list. Within each list type, one group recalled the list at $2 \mathrm{~min}$. after learning, one group at $45 \mathrm{~min}$., and one group at 1 week.

The low-arousal list showed high immediate recall which decreased with time. The higharousal list showed some rise in recall with time. Other analyses agreed with the results of Kleinsmith and Kaplan (1963b) showing high immediate and low ultimate recall for items learned under low arousal, and low immediate recall and high ultimate recall for items learned under high arousal.

Analyses are presented to account for the strong recency effect in the serial position curve for 2-min. recall in terms of short-term memory, and to account for the strong primacy effect in the one week recall serial position curve on the basis of arousal by serial position.

Kueinsmith, L. J., and Kaplan, S. Paired associates learning as a function of arousal and interpolated interval. J. exp. Psychol., 1963, 65, 190-193. (a)

KLeInsmith, L. J., and Kaplan, S. The interaction of arousal and recall interval in nonsense syllable paired associates learning. J. exp. Psychol., 1963 , in press. (b)

LykKen, D. T. Properties of electrodes used in electrodermal measurements. J. comp. physiol. Psychol., 1959, 52, 629-634.

Melton, A. W. Implications of short-term memory for a general theory of memory. J. verbal Learn. verbal Behav., 1963, 2 (in this issue). 
WALKER, E. L. The duration and course of reaction decrement and the influence of reward. J. comp. physiol. Psychol., 1956, 49, 167-176.

WALKER, E. L. Action decrement and its relation to learning. Psychol. Rev., 1958, 65, 129-142.

WaLker, E. L., AND Motoyoshr, R. The effects of amount of reward and distribution of practice on active and inactive memory traces. $J$. comp. physiol. Psychol., 1962, 55, 32-36.

Walker, E. L., and Paradise, N. A positive correlation between action decrement and learning. J. exp. Psychol., 1958, 56, 45-47.

(Received March 21，1963) 